\title{
Random Procedures for Dominating Sets in Graphs
}

\author{
Sarah Artmann \\ Institut für Mathematik \\ TU Ilmenau, D-98684 Ilmenau, Germany \\ sarah.artmann@tu-ilmenau.de \\ Frank Göring \\ Fakultät für Mathematik \\ TU Chemnitz, D-09107 Chemnitz, Germany \\ frank.goering@mathematik.tu-chemnitz.de \\ Jochen Harant \\ Institut für Mathematik \\ TU Ilmenau, D-98684 Ilmenau, Germany \\ jochen.harant@tu-ilmenau.de \\ Dieter Rautenbach \\ Institut für Optimierung und Operations Research \\ Universität Ulm, D-89069 Ulm, Germany \\ dieter.rautenbach@uni-ulm.de

\section{Ingo Schiermeyer}

Institut für Diskrete Mathematik und Algebra

TU Bergakademie Freiberg, D-09596 Freiberg, Germany

Ingo.Schiermeyer@math.tu-freiberg. de

Submitted: Jun 26, 2008; Accepted: Jul 13, 2010; Published: Jul 20, 2010

Mathematics Subject Classifications: 05C69

\begin{abstract}
We present and analyze some random procedures for the construction of small dominating sets in graphs. Several upper bounds for the domination number of a graph are derived from these procedures.
\end{abstract}

Keywords: domination; independence; probabilistic method 


\section{Introduction}

We consider finite, simple and undirected graphs $G=(V, E)$ with vertex set $V$, edge set $E$, order $n=|V|$, and size $m=|E|$. The neighbourhood of a vertex $u \in V$ in the graph $G$ is the set $N_{G}(u)=\{v \in V \mid u v \in E\}$ and the closed neighbourhood of $u$ in $G$ is $N_{G}[u]=N_{G}(u) \cup\{u\}$. The degree of $u$ in $G$ is the number $d_{G}(u)=\left|N_{G}(u)\right|$ of its neighbours. For a set $U \subseteq V$ let $N_{G}[U]=\bigcup_{u \in U} N_{G}[u]$ and $N_{G}(U)=N_{G}[U] \backslash U$.

A set of vertices $D \subseteq V$ of $G$ is dominating, if every vertex in $V \backslash D$ has a neighbour in $D$. The minimum cardinality of a dominating set is the domination number $\gamma(G)$ of $G$. A set of vertices $I \subseteq V$ of $G$ is independent, if no two vertices in $I$ are adjacent. The maximum cardinality of an independent set is the independence number $\alpha(G)$ of $G$.

Dominating and independent sets are among the most well-studied graph theoretical objects. The literature on this subject has been surveyed and detailed in the two books by Haynes, Hedetniemi, and Slater [7, 8]. Natural conditions used to obtain upper bounds on the domination number involve the order of the considered graphs and the degrees of their vertices or just their minimum degree. While there are several results for small minimum degrees $[9,10,12]$, asymptotically best-possible bounds in terms of the order and the minimum degree can be obtained by very simple probabilistic arguments [1] (cf. also $[2,11])$.

In the present paper we analyze random procedures for the construction of dominating sets in more detail. In Section 2 we generalize the argument from Alon and Spencer [1] which works in two rounds to several rounds. As observed in Section 3 several random procedures lead to bounds involving multilinear functions and the partial derivaties of these functions can be used to improve the bounds. Finally, in Section 4 we propose a new procedure for the construction of dominating sets which mimics a beautiful probabilistic argument for Caro and Wei's lower bound on the independence number $[4,13]$.

\section{Constructing a Dominating Set in several Rounds}

A very simple probabilistic argument due to Alon and Spencer [1] implies that for every graph $G$ of order $n$ and minimum degree $\delta$ the domination number satisfies

$$
\gamma(G) \leqslant \frac{\ln (\delta+1)+1}{\delta+1} n
$$

which is asymptotically best-possible with respect to the dependence on $\delta$. They construct a dominating set in two steps. They first select a set $X$ of vertices containing every vertex of $G$ independently at random with probability $p$ and then they add the set $R$ of vertices of $G$ which are not yet dominated, i.e. $R=V \backslash N_{G}[X]$. The bound on the domination number is obtained by estimating the expected cardinality of the dominating set $X \cup R$ in terms of $p$ and optimizing over $p \in[0,1]$.

Here we consider a generalization of this approach which works in several rounds. A first natural idea would be to select a random set of vertices, a second random set of vertices among those vertices which are still not dominated by the first set, a third 
random set of vertices among those vertices which are still not dominated by the first two sets and so on. The problem with this approach is that the involved probabilities are hard to analyze because of accumulating dependencies. Therefore, we modify this idea as follows. We select $k$ sets of vertices $X_{1}, \ldots, X_{k}$ independently at random. Now for every $i=1, \ldots, k$ the set $Y_{i}$ will contain the vertices from $X_{i}$ which are not yet dominated by $X_{1} \cup \cdots \cup X_{i-1}$, i.e. $Y_{i}$ will in fact be similar to the sets described above. To avoid dependencies we add to $Y_{i}$ a set $Z_{i}$ ensuring that $\left(Y_{1} \cup Z_{1}\right) \cup \cdots\left(Y_{i} \cup Z_{i}\right)$ dominates all vertices dominated by $X_{1} \cup \cdots \cup X_{i}$. To make the analysis possible we still need to assume that the graph has no cycles of length less than five, i.e. its girth is at least five.

Theorem 1 Let $G=(V, E)$ be a graph of maximum degree $\Delta$ and girth at least five. For some $k \in \mathbb{N}$ let $p_{1}, \ldots, p_{k} \in[0,1]$. If $p_{<1}=0$ and $p_{<i}=1-\prod_{j=1}^{i-1}\left(1-p_{j}\right)$ for $2 \leqslant i \leqslant k$, then

$$
\begin{aligned}
\gamma(G) \leqslant & \sum_{v \in V}\left(\sum_{i=1}^{k} p_{i} \cdot\left(1-p_{<i}\right)^{\left(d_{G}(v)+1\right)}\right. \\
& +\sum_{i=1}^{k-1}\left(1-p_{<i}\right)^{\left(d_{G}(v)+1\right)} \cdot\left(1-p_{i}\right) \cdot\left(\left(1-p_{i}\left(1-p_{<i}\right)^{(\Delta-1)}\right)^{d_{G}(v)}-\left(1-p_{i}\right)^{d_{G}(v)}\right) \\
& \left.+\left(1-p_{<k}\right)^{\left(d_{G}(v)+1\right)} \cdot\left(1-p_{k}\right) \cdot\left(1-p_{k}\left(1-p_{<k}\right)^{(\Delta-1)}\right)^{d_{G}(v)}\right) .
\end{aligned}
$$

Proof: For $1 \leqslant i \leqslant k$ let $X_{i}$ be a subset of $V$ which arises by choosing every vertex of $G$ independently at random with probability $p_{i}$. Let $Y_{1}=X_{1}$ and $Z_{1}=\emptyset$. For $2 \leqslant i \leqslant k$ let

$$
\begin{gathered}
X_{<i}=\bigcup_{j=1}^{i-1} X_{j}, \\
Y_{i}=X_{i} \backslash N_{G}\left[X_{<i}\right]
\end{gathered}
$$

and

$$
Z_{i}=N_{G}\left[X_{i}\right] \backslash N_{G}\left[X_{<i} \cup Y_{i}\right]
$$

Let

$$
R=V \backslash N_{G}\left[\bigcup_{j=1}^{k} X_{j}\right] .
$$

Claim $1 N_{G}\left[X_{1} \cup \cdots \cup X_{i}\right] \subseteq N_{G}\left[\left(Y_{1} \cup Z_{1}\right) \cup \cdots \cup\left(Y_{i} \cup Z_{i}\right)\right]$ for $1 \leqslant i \leqslant k$.

Proof of Claim 1: We prove the claim by induction. For $i=1$ the statement is trivial, since $X_{1}=Y_{1} \cup Z_{1}$. Now let $i \geqslant 2$. By induction, $N_{G}\left[X_{<i}\right] \subseteq N_{G}\left[\left(Y_{1} \cup Z_{1}\right) \cup \cdots \cup\left(Y_{i} \cup Z_{i}\right)\right]$ and it suffices to show $N_{G}\left[X_{i}\right] \subseteq N_{G}\left[\left(Y_{1} \cup Z_{1}\right) \cup \cdots \cup\left(Y_{i} \cup Z_{i}\right)\right]$. Let $v \in N_{G}\left[X_{i}\right]$.

If $v \in X_{i}$, then either $v \in Y_{i}$ or $v \in N_{G}\left[X_{<i}\right]$. In both cases we are done. If $v \in N_{G}\left(X_{i}\right)$, then either $v \in N_{G}\left[X_{<i}\right]$ or $v \in N_{G}\left[Y_{i}\right]$ or, by definition, $v \in Z_{i}$. Again, in all cases we are done and the proof of the claim is complete. 
Note that, by the claim and the definition of $R$, the set

$$
D=R \cup \bigcup_{i=1}^{k}\left(Y_{i} \cup Z_{i}\right)
$$

is a dominating set of $G$.

The expected cardinality of $Y_{1}$ is $p_{1} n$. Now let $2 \leqslant i \leqslant k$. Since the sets $X_{1}, \ldots, X_{i-1}$ are chosen independently, the set $X_{<i}$ arises by choosing every vertex of $G$ independently at random with probability

$$
p_{<i}=1-\prod_{j=1}^{i-1}\left(1-p_{j}\right)
$$

Hence

$$
\mathbf{P}\left[v \in Y_{i}\right]=p_{i} \cdot\left(1-p_{<i}\right)^{\left(d_{G}(v)+1\right)}
$$

for every vertex $v \in V$.

Furthermore, a vertex $v \in V$ is in $Z_{i}$ if and only if $v \notin N_{G}\left[X_{<i}\right]$ and $v \notin X_{i}$ and there is some non-empty set $U \subseteq N_{G}(v)$ with $N_{G}(v) \cap\left(N_{G}\left(X_{<i}\right) \cap X_{i}\right)=U$ and $N_{G}(v) \cap\left(V \backslash X_{i}\right)=$ $N_{G}(v) \backslash U$.

For some specific set $U$ let

$$
N_{G}(v) \backslash U=\left\{v_{1}, v_{2}, \ldots, v_{d_{G}(v)-l}\right\}
$$

and

$$
U=\left\{v_{d_{G}(v)-l+1}, v_{d_{G}(v)-l+2}, \ldots, v_{d_{G}(v)}\right\} .
$$

By the independence of the choice of the elements of the sets $X_{j}$ and by the girth condition, we obtain - in what follows we indicate the use of the independence by "(i)" and the use of the girth condition by "(g)"

$$
\begin{aligned}
& \mathbf{P}\left[v \in Z_{i} \mid\left(N_{G}(v) \cap\left(N_{G}\left(X_{<i}\right) \cap X_{i}\right)=U\right) \wedge\left(N_{G}(v) \cap\left(V \backslash X_{i}\right)=N_{G}(v) \backslash U\right)\right] \\
= & \mathbf{P}\left[\left(v \notin N_{G}\left[X_{<i}\right]\right) \wedge\left(v \notin X_{i}\right) \wedge\left(\bigwedge_{j=1}^{d_{G}(v)-l}\left(v_{j} \notin X_{i}\right)\right) \wedge\left(\bigwedge_{j=d_{G}(v)-l+1}^{d_{G}(v)}\left(v_{j} \in N_{G}\left(X_{<i}\right) \cap X_{i}\right)\right)\right] \\
\stackrel{(i)}{=} & \left(1-p_{<i}\right)^{\left(d_{G}(v)+1\right)} \cdot\left(1-p_{i}\right) \cdot\left(1-p_{i}\right)^{\left(d_{G}(v)-l\right)} \\
& \left.\cdot \mathbf{P}\left[\bigwedge_{j=d_{G}(v)-l+1}^{d_{G}(v)}\left(v_{j} \in N_{G}\left(X_{<i}\right) \cap X_{i}\right)\right) \mid\left(v \notin N_{G}\left[X_{<i}\right]\right) \wedge\left(v \notin X_{i}\right) \wedge\left(\bigwedge_{j=1}^{d_{G}(v)-l}\left(v_{j} \notin X_{i}\right)\right)\right] \\
\stackrel{(i)}{=} & \left(1-p_{<i}\right)^{\left(d_{G}(v)+1\right)} \cdot\left(1-p_{i}\right)^{\left(d_{G}(v)-l+1\right)} \\
& \cdot \mathbf{P}\left[\left(\bigwedge_{d_{G}(v)}\left(\bigwedge_{j} \in N_{G}\left(X_{<i}\right) \cap X_{i}\right)\right) \mid v \notin N_{G}\left[X_{<i}\right]\right]
\end{aligned}
$$




$$
\begin{aligned}
& =\left(1-p_{<i}\right)^{\left(d_{G}(v)+1\right)} \cdot\left(1-p_{i}\right)^{\left(d_{G}(v)-l+1\right)} \\
& \cdot \prod_{j=d_{G}(v)-l+1}^{d_{G}(v)} \mathbf{P}\left[\left(v_{j} \in N_{G}\left(X_{<i}\right) \cap X_{i}\right) \mid\left(\bigwedge_{r=d_{G}(v)-l+1}^{j-1}\left(v_{r} \in N_{G}\left(X_{<i}\right) \cap X_{i}\right)\right) \wedge\left(v \notin N_{G}\left[X_{<i}\right]\right)\right] \\
& \stackrel{(i)}{=}\left(1-p_{<i}\right)^{\left(d_{G}(v)+1\right)} \cdot\left(1-p_{i}\right)^{\left(d_{G}(v)-l+1\right)} \\
& \cdot p_{i}^{l} \cdot \prod_{j=d_{G}(v)-l+1}^{d_{G}(v)} \mathbf{P}\left[\left(v_{j} \in N_{G}\left(X_{<i}\right)\right) \mid\left(\bigwedge_{r=d_{G}(v)-l+1}^{j-1}\left(v_{r} \in N_{G}\left(X_{<i}\right)\right)\right) \wedge\left(v \notin N_{G}\left[X_{<i}\right]\right)\right] \\
& \stackrel{(\underline{g})}{=}\left(1-p_{<i}\right)^{\left(d_{G}(v)+1\right)} \cdot\left(1-p_{i}\right)^{\left(d_{G}(v)-l+1\right)} \cdot p_{i}^{l} \cdot \prod_{j=d_{G}(v)-l+1}^{d_{G}(v)} \mathbf{P}\left[\left(v_{j} \in N_{G}\left(X_{<i}\right)\right) \mid v \notin N_{G}\left[X_{<i}\right]\right] \\
& \stackrel{(g)}{=}\left(1-p_{<i}\right)^{\left(d_{G}(v)+1\right)} \cdot\left(1-p_{i}\right)^{\left(d_{G}(v)-l+1\right)} \cdot p_{i}^{l} \cdot \prod_{j=d_{G}(v)-l+1}^{d_{G}(v)}\left(1-\left(1-p_{<i}\right)^{\left(d_{G}\left(v_{j}\right)-1\right)}\right) . \\
& \leqslant\left(1-p_{<i}\right)^{\left(d_{G}(v)+1\right)} \cdot\left(1-p_{i}\right)^{\left(d_{G}(v)-l+1\right)} \cdot p_{i}^{l} \cdot\left(1-\left(1-p_{<i}\right)^{(\Delta-1)}\right)^{l} .
\end{aligned}
$$

This implies that

$$
\begin{aligned}
& \mathbf{P}\left[v \in Z_{i}\right] \\
\leqslant & \left(1-p_{<i}\right)^{\left(d_{G}(v)+1\right)} \cdot\left(1-p_{i}\right) \cdot \sum_{l=1}^{d_{G}(v)}\left(\begin{array}{c}
d_{G}(v) \\
l
\end{array}\right) \cdot\left(1-p_{i}\right)^{\left(d_{G}(v)-l\right)} \cdot p_{i}^{l} \cdot\left(1-\left(1-p_{<i}\right)^{(\Delta-1)}\right)^{l} \\
= & \left(1-p_{<i}\right)^{\left(d_{G}(v)+1\right)} \cdot\left(1-p_{i}\right) \cdot\left(\left(\left(1-p_{i}\right)+p_{i}\left(1-\left(1-p_{<i}\right)^{(\Delta-1)}\right)\right)^{d_{G}(v)}-\left(1-p_{i}\right)^{d_{G}(v)}\right) \\
= & \left(1-p_{<i}\right)^{\left(d_{G}(v)+1\right)} \cdot\left(1-p_{i}\right) \cdot\left(\left(1-p_{i}\left(1-p_{<i}\right)^{(\Delta-1)}\right)^{d_{G}(v)}-\left(1-p_{i}\right)^{d_{G}(v)}\right)
\end{aligned}
$$

for every vertex $v \in V$.

Finally,

$$
\mathbf{P}[v \in R]=\prod_{i=1}^{k}\left(1-p_{i}\right)^{\left(d_{G}(v)+1\right)}
$$

for every vertex $v \in V$. 
By linearity of expectation, we obtain

$$
\begin{aligned}
\gamma(G) \leqslant & \mathbf{E}[|D|] \\
= & \mathbf{E}[|R|]+\sum_{i=1}^{k}\left(\mathbf{E}\left[\left|Y_{i}\right|\right]+\mathbf{E}\left[\left|Z_{i}\right|\right]\right) \\
\leqslant & \sum_{v \in V}\left(\prod_{i=1}^{k}\left(1-p_{i}\right)^{\left(d_{G}(v)+1\right)}+\sum_{i=1}^{k} p_{i} \cdot\left(1-p_{<i}\right)^{\left(d_{G}(v)+1\right)}\right. \\
& \left.+\sum_{i=1}^{k}\left(1-p_{<i}\right)^{\left(d_{G}(v)+1\right)} \cdot\left(1-p_{i}\right) \cdot\left(\left(1-p_{i}\left(1-p_{<i}\right)^{(\Delta-1)}\right)^{d_{G}(v)}-\left(1-p_{i}\right)^{d_{G}(v)}\right)\right) \\
= & \sum_{v \in V}\left(\sum_{i=1}^{k} p_{i} \cdot\left(1-p_{<i}\right)^{\left(d_{G}(v)+1\right)}\right. \\
& +\sum_{i=1}^{k-1}\left(1-p_{<i}\right)^{\left(d_{G}(v)+1\right)} \cdot\left(1-p_{i}\right) \cdot\left(\left(1-p_{i}\left(1-p_{<i}\right)^{(\Delta-1)}\right)^{d_{G}(v)}-\left(1-p_{i}\right)^{d_{G}(v)}\right) \\
& \left.+\left(1-p_{<k}\right)^{\left(d_{G}(v)+1\right)} \cdot\left(1-p_{k}\right) \cdot\left(1-p_{k}\left(1-p_{<k}\right)^{(\Delta-1)}\right)^{d_{G}(v)}\right)
\end{aligned}
$$

and the proof is complete.

Theorem 1 still leaves the task to find good values for the probabilites $p_{1}, \ldots, p_{k}$. In order to compare it for instance to the bound (1) of Alon and Spencer, we present some numerical results for $d$-regular graphs and different numbers of rounds. Table 1 gives the numerically optimal value for the bound on $\frac{\gamma(G)}{|V|}$ in Theorem 1 for $3 \leqslant d \leqslant 10$ and $1,2,3$ and 11 rounds. For comparision we also list the value of (1).

\begin{tabular}{|r||c||c|c|c|c|}
\hline \multicolumn{1}{|c||}{} & \multicolumn{4}{||}{} & \multicolumn{4}{c|}{ Rounds } \\
\hline $\mathrm{d}$ & $\frac{\ln (d+1)+1}{d+1}$ & 1 & 2 & 3 & 11 \\
\hline \hline 3 & 0.59657359028 & 0.52752960628 & 0.46398402832 & 0.45378488660 & 0.45258151834 \\
4 & 0.52188758248 & 0.46500775601 & 0.40965805121 & 0.40614010876 & 0.40609337873 \\
5 & 0.46529324487 & 0.41764406769 & 0.36881380436 & 0.36756994127 & 0.36756737023 \\
6 & 0.42084430700 & 0.38026854880 & 0.33667455575 & 0.33620842585 & 0.33620824046 \\
7 & 0.38493019271 & 0.34987749850 & 0.31055501904 & 0.31037371778 & 0.31037370239 \\
8 & 0.35524717526 & 0.32459050164 & 0.28880727138 & 0.28873522218 & 0.28873522080 \\
9 & 0.33025850929 & 0.30316268558 & 0.27035398149 & 0.27032500642 & 0.27032500629 \\
10 & 0.30889957025 & 0.28473323436 & 0.25445619977 & 0.25444447470 & 0.25444447469 \\
\hline
\end{tabular}

Table 1 Numerical results for Theorem 1

For the results using 11 rounds the numerically optimal $p_{i}$ 's are listed in Table 2. 


\begin{tabular}{|r||c|c|c|c|}
\hline \multicolumn{1}{|c||}{} & \multicolumn{4}{|c|}{ Degree of regularity $d$} \\
\hline $\mathrm{i}$ & 3 & 4 & 5 & 10 \\
\hline \hline 1 & 0.15802495270865785 & 0.17961282083328788 & 0.17625843720156733 & 0.13613621200382378 \\
2 & 0.26758130289201026 & 0.34475712015729920 & 0.36944988288580227 & 0.37255216737900287 \\
3 & 0.37728274633574865 & 0.45530927158279288 & 0.47802072348063751 & 0.49999885780393971 \\
4 & 0.43639455423559259 & 0.48557411477730633 & 0.49999501914405736 & 0.49999999999999550 \\
5 & 0.45789313248767055 & 0.49996125731020485 & 0.49999999660914201 & 0.50000000000000000 \\
6 & 0.46463706700970097 & 0.49999985782508249 & 0.49999999999677913 & 0.50000000000000000 \\
7 & 0.49946145125621827 & 0.49999999944911738 & 0.49999999999999683 & 0.50000000000000000 \\
8 & 0.49999169039055640 & 0.49999999999785022 & 0.50000000000000000 & 0.50000000000000000 \\
9 & 0.49999987061638329 & 0.4999999999999161 & 0.50000000000000000 & 0.50000000000000000 \\
10 & 0.49999999801110439 & 0.5000000000000000 & 0.50000000000000000 & 0.50000000000000000 \\
11 & 0.49999999999999789 & 0.50000000000000000 & 0.50000000000000000 & 0.50000000000000000 \\
\hline
\end{tabular}

Table 2 Optimal choices for the $p_{i}$ 's

\section{Optimizing the Results of Random Procedures}

Many random procedures constructing dominating sets essentially yield a bound on the domination number in terms of a multilinear function depending on the involved probabilities. For instance, if we use an individual probability $p_{u}$ for every vertex $u \in V$ of the graph $G=(V, E)$ in the procedure of Alon and Spencer [1], then the expected cardinality of the resulting dominating set equals $\sum_{u \in V}\left(p_{u}+\prod_{v \in N_{G}[u]}\left(1-p_{v}\right)\right)$. This is in fact a multilinear function, i.e. fixing all but one variable results in a linear function.

To obtain a compact expression as a bound one often sets all values of $p_{u}$ equal to some $p$ and solves the arising one-dimensional optimization problem over $p \in[0,1]$.

Here we propose a modification of this approach. Given values for the probabilities $p_{u}$ the partial derivatives of the multilinear bound indicate changes of the $p_{u}$ which would decrease the value of the bound. Depending on the partial derivatives we will reset the $p_{u}$ to 0 or 1 . To allow for some further flexibility we use a parameter $b$ in order to decide which values to modify in which way.

Given a multilinear function $f\left(x_{1}, \ldots, x_{n}\right)$, some $x \in[0,1]$, and some $b \geqslant 0$ consider the following algorithm $A_{b}(x)$.

Algorihm $A_{b}(x)$

1. For $i$ from 1 to $n$ do: $x_{i}:=x$.

2. For $i$ from 1 to $n$ do: if $f_{x_{i}}\left(x_{1}, \ldots, x_{n}\right)>-b$, then $x_{i}:=0$, else $x_{i}:=1$.

3. For $i$ from 1 to $n$ do: if $f_{x_{i}}\left(x_{1}, \ldots, x_{n}\right) \leqslant-b$, then $x_{i}:=1$.

4. Output $\left(x_{1}, \ldots, x_{n}\right)$. 
Theorem 2 Let $G=(V, E)$ be a graph with vertex set $V=\left\{v_{1}, v_{2}, \ldots, v_{n}\right\}$ and minimum degree $\delta$. Let $f\left(x_{1}, \ldots, x_{n}\right)$ be a multilinear function such that

$$
\gamma(G) \leqslant \min _{\left(x_{1}, \ldots, x_{n}\right) \in[0,1]^{n}} f\left(x_{1}, \ldots, x_{n}\right) .
$$

Furthermore, for some $b \geqslant 0$ and every $x \in[0,1]$ let the Algorithm $A_{b}(x)$ produce a vector $\left(x_{1}, x_{2}, \ldots, x_{n}\right)$ with the property that $x_{k}=0$ for all $1 \leqslant k \leqslant n$ with $v_{k} \in N_{G}\left[v_{i}\right] \cup N_{G}\left[v_{j}\right]$ for some $1 \leqslant i<j \leqslant n$ implies $\operatorname{dist}_{G}\left(v_{i}, v_{j}\right) \geqslant 3$.

Then

$$
\gamma(G) \leqslant \min _{x \in[0,1]}\left(\frac{\delta}{\delta(1+b)+b} f(x, \ldots, x)+\frac{b(\delta x+1)}{\delta(1+b)+b} n\right) .
$$

Before we proceed to the proof of Theorem 2 we introduce some terminology. Given the situation described in Theorem 2 we will call a vertex $v_{i} \in V$ critical, if $x_{k}=0$ for all $1 \leqslant k \leqslant n$ with $v_{k} \in N_{G}\left[v_{i}\right]$. The property described in Theorem 2 means that Algorithm $A_{b}(x)$ produces a vector $\left(x_{1}, x_{2}, \ldots, x_{n}\right)$ for which the critical vertices have pairwise distance at least three. If the function $f$ - associated to the graph $G$ - has this property, then we say that $f$ has property $\mathcal{P}_{b}$.

Proof of Theorem 2: Let $G, b$ and $f$ be as in the statement of Theorem 2 .

Since $f$ is multilinear, we have for all $x_{1}, \ldots, x_{n}, \delta x_{i} \in \mathbb{R}$

$$
\begin{aligned}
& f\left(x_{1}, \ldots, x_{i-1}, x_{i}+\delta x_{i}, x_{i+1}, \ldots, x_{n}\right) \\
= & f\left(x_{1}, \ldots, x_{i-1}, x_{i}, x_{i+1}, \ldots, x_{n}\right)+\frac{\partial}{\partial x_{i}} f\left(x_{1}, \ldots, x_{i-1}, x_{i}, x_{i+1}, \ldots, x_{n}\right) \cdot \delta x_{i} .
\end{aligned}
$$

For some $x \in[0,1]$ let $\left(x_{1}, \ldots, x_{n}\right)$ denote the output of Algorithm $A_{b}(x)$. Let

$$
M=\left\{v_{i} \in V(G) \mid x_{i}=1\right\} .
$$

Note that a vertex $v_{i}$ is critical exactly if $N_{G}\left[v_{i}\right] \cap M=\emptyset$.

Claim $1 \gamma(G) \leqslant f(x, \ldots, x)-b|M|+b x n$.

Proof of Claim 1: By $(2), \gamma(G) \leqslant f(x, \ldots, x)$. We consider the Algorithm $A_{b}(x)$. After Step $1,\left(x_{1}, \ldots, x_{n}\right)=(x, \ldots, x)$. If during Step 2 some $x_{i}=x$ is replaced by 1 , then, by (3), the value of $f\left(x_{1}, \ldots, x_{n}\right)$ decreases at least by $b(1-x)$. Similarly, if during Step 2 some $x_{i}=x$ is replaced by 0 , then, by $(3)$, the value of $f\left(x_{1}, \ldots, x_{n}\right)$ increases at most by $b x$. Furthermore, if during Step 3 some $x_{i}=0$ is replaced by 1 , then $x_{i}=x$ was replaced by 0 in Step 2 and summing the effect of the changes in $x_{i}$ made by Step 2 and Step 3, $f\left(x_{1}, \ldots, x_{n}\right)$ decreases at least by $b(1-x)$ in total. Altogether,

$$
f\left(x_{1}, \ldots, x_{n}\right) \leqslant f(x, \ldots, x)-b(1-x)|M|+b x(n-|M|)=f(x, \ldots, x)-b|M|+b x n .
$$

which completes the proof of the claim. 
Let $k$ be the number of critical vertices and let $D$ be obtained by adding all critical vertices to $M$. Clearly, $D$ is a dominating set of $G, \gamma(G) \leqslant|D|=|M|+k$, and, by Claim 1 ,

$$
\begin{aligned}
\gamma(G) & =\left(\frac{1}{1+b}+\frac{b}{1+b}\right) \gamma(G) \\
& \leqslant \frac{1}{1+b}(f(x, \ldots, x)-b|M|+b x n)+\frac{b}{1+b}|D| \\
& =\frac{1}{1+b}(f(x, \ldots, x)-b(|D|-k)+b x n)+\frac{b}{1+b}|D| \\
& =\frac{1}{1+b} f(x, \ldots, x)+\frac{b}{1+b}(k+x n) .
\end{aligned}
$$

Since $f$ has property $\mathcal{P}_{b}$,

$$
\gamma(G) \leqslant n-\delta k
$$

Since $\frac{\delta(1+b)}{\delta(1+b)+b}+\frac{b}{\delta(1+b)+b}=1$, a convex combination of (4) and (5) yields

$$
\begin{aligned}
\gamma(G) & \leqslant \frac{\delta(1+b)}{\delta(1+b)+b}\left(\frac{1}{1+b} f(x, \ldots, x)+\frac{b}{1+b}(k+x n)\right)+\frac{b}{\delta(1+b)+b}(n-\delta k) \\
& =\frac{\delta}{\delta(1+b)+b} f(x, \ldots, x)+\frac{b(\delta x+1)}{\delta(1+b)+b} n .
\end{aligned}
$$

Since $x$ was arbitrary in $[0,1]$, the theorem follows.

We will now show an application of Theorem 2. Our next lemma gives an upper bound on the domination number in terms of a multilinear function as required for Theorem 2 (similar bounds are contained in [5]). Additionally we have to verify property $\mathcal{P}_{b}$ for some $b$.

Proposition 3 If $G=(V, E)$ is a graph with vertex set $V=\left\{v_{1}, \ldots, v_{n}\right\}$ and without isolated vertices, then

$$
\gamma(G)=\min _{\left(x_{1}, \ldots, x_{n}\right) \in[0,1]^{n}} f\left(x_{1}, \ldots, x_{n}\right)
$$

where

$$
f\left(x_{1}, \ldots, x_{n}\right)=\sum_{i=1}^{n}\left(x_{i}+\prod_{v_{j} \in N_{G}\left[v_{i}\right]}\left(1-x_{j}\right)-\frac{1}{1+d_{G}\left(v_{i}\right)} \prod_{v_{j} \in N_{G}\left[v_{i}\right]} x_{j}\right) .
$$

Furthermore, the function $f$ in (7) has property $\mathcal{P}_{1}$.

Proof: Let $\left(x_{1}, \ldots, x_{n}\right) \in[0,1]^{n}$ and let $X \subseteq V$ be a set of vertices containing every vertex $v_{i}$ independently at random with probability $x_{i}$. Let

$$
X^{\prime}=\left\{v_{i} \in V \mid N_{G}\left[v_{i}\right] \subseteq X\right\}
$$


and let $I$ be a maximum independent set in the subgraph $G\left[X^{\prime}\right]$ of $G$ induced by $X^{\prime}$. If

$$
Y=\left\{v \in V \mid N_{G}[v] \cap X=\emptyset\right\}
$$

then $(X \backslash I) \cup Y$ is a dominating set of $G$ and hence $\gamma(G) \leqslant \mathbf{E}[|X|]+\mathbf{E}[|Y|]-\mathbf{E}[|I|]$. Clearly, $\mathbf{E}[|X|]=\sum_{i=1}^{n} x_{i}$ and $\mathbf{E}[|Y|]=\sum_{i=1}^{n} \prod_{v_{j} \in N_{G}\left[v_{i}\right]}\left(1-x_{j}\right)$.

By the Caro-Wei inequality [4, 13],

$$
\mathbf{E}[|I|] \geqslant \sum_{v \in X^{\prime}} \frac{1}{1+d_{G\left[X^{\prime}\right]}(v)} \geqslant \sum_{v \in V} \frac{1}{1+d_{G}(v)} \mathbf{P}\left[v \in X^{\prime}\right]=\sum_{i=1}^{n} \frac{1}{1+d_{G}\left(v_{i}\right)} \prod_{v_{j} \in N_{G}\left[v_{i}\right]} x_{j} .
$$

This implies that $\gamma(G)$ is at most the expression given on the right hand side of (6). For the converse, let $D$ be a minimum dominating set. Note that for every vertex $v_{i} \in V$ we have $N_{G}\left[v_{i}\right] \cap D \neq \emptyset$, since $D$ is dominating and $N_{G}\left[v_{i}\right] \cap D \neq N_{G}\left[v_{i}\right]$, since $D$ is minimum. Therefore, setting $x_{i}^{*}=1$ for all $v_{i} \in D$ and $x_{i}^{*}=0$ for all $v_{i} \in V \backslash D$ yields

$$
\begin{aligned}
\gamma(G) & =\sum_{i=1}^{n}\left(x_{i}^{*}+\prod_{v_{j} \in N_{G}\left[v_{i}\right]}\left(1-x_{j}^{*}\right)-\frac{1}{1+d_{G}\left(v_{i}\right)} \prod_{v_{j} \in N_{G}\left[v_{i}\right]} x_{j}^{*}\right) \\
& =\sum_{i=1}^{n}\left(x_{i}^{*}+0+0\right)=|D|=\gamma(G)
\end{aligned}
$$

and the proof of (6) is complete.

Now we proceed to the proof that $f$ has property $\mathcal{P}_{1}$. Therefore, let $x \in[0,1]$, let $\left(x_{1}, \ldots, x_{n}\right)$ be the output of Algorithm $A_{1}(x)$ and let $v_{i}$ and $v_{j}$ be two critical vertices. For contradiction, we assume that $N_{G}\left[v_{i}\right] \cap N_{G}\left[v_{j}\right] \neq \emptyset$. Note that after the execution of Step 2 the values $x_{l}$ for all $v_{l} \in N_{G}\left[v_{i}\right] \cup N_{G}\left[v_{j}\right]$ are 0 and remain 0 throughout the execution of Step 3. For $1 \leqslant k \leqslant n$ we have

$$
\begin{aligned}
& \frac{\partial}{\partial x_{k}} f\left(x_{1}, \ldots, x_{n}\right) \\
= & 1-\sum_{v_{l} \in N_{G}\left[v_{k}\right]}\left(\prod_{v_{m} \in N_{G}\left[v_{l}\right] \backslash\left\{v_{k}\right\}}\left(1-v_{x}\right)+\frac{1}{1+d_{G}\left(v_{l}\right)} \prod_{v_{m} \in N_{G}\left[v_{l}\right] \backslash\left\{v_{k}\right\}} x_{m}\right) .
\end{aligned}
$$

If $v_{j} \in N_{G}\left[v_{i}\right]$, then during the execution of Step 3

$$
\frac{\partial}{\partial x_{i}} f\left(x_{1}, \ldots, x_{n}\right) \leqslant 1-\prod_{v_{m} \in N_{G}\left[v_{i}\right] \backslash\left\{v_{i}\right\}}\left(1-x_{m}\right)-\prod_{v_{m} \in N_{G}\left[v_{j}\right] \backslash\left\{v_{i}\right\}}\left(1-x_{m}\right)=-1
$$

and if $v_{k} \in N_{G}\left(v_{i}\right) \cap N_{G}\left(v_{j}\right)$, then during the execution of Step 3

$$
\frac{\partial}{\partial x_{k}} f\left(x_{1}, \ldots, x_{n}\right) \leqslant 1-\prod_{v_{m} \in N_{G}\left[v_{i}\right] \backslash\left\{v_{k}\right\}}\left(1-x_{m}\right)-\prod_{v_{m} \in N_{G}\left[v_{j}\right] \backslash\left\{v_{k}\right\}}\left(1-x_{m}\right)=-1 .
$$


In both cases, we obtain the contradiction that either $x_{i}$ or $x_{k}$ would be set to 1 by Step 3 and the proof is complete.

Theorem 2 and Proposition 3 immediately imply the following result for $b=1$.

Corollary 4 If $G=(V, E)$ is a graph of order $n$ and minimum degree $\delta$, then

$$
\gamma(G) \leqslant \frac{1}{2 \delta+1}\left((2 \delta x+1) n+\delta \sum_{v \in V}\left((1-x)^{d_{G}(v)+1}-\frac{1}{1+d_{G}(v)} x^{d_{G}(v)+1}\right)\right)
$$

for every $x \in[0,1]$.

For $\delta \geqslant 3$ we can derive the following bound.

Corollary 5 Let $G$ be a graph of order $n$ and minimum degree $\delta \geqslant 3$. The equation $(\delta+1)(1-x)^{\delta}+x^{\delta}=2$ has a unique solution $x_{0} \in\left[0, \frac{1}{2}\right]$ for which

$$
\frac{\gamma(G)}{n} \leqslant \frac{1}{2 \delta+1}\left(\left(2 \delta x_{0}+1\right)+\delta\left(\left(1-x_{0}\right)^{\delta+1}-\frac{1}{1+\delta} x_{0}^{\delta+1}\right)\right) .
$$

Proof: We will first show that the contribution of every vertex to the bound in Corollary 4 decreases monotonously with its degree provided that $x$ is within a certain range.

Claim 1 If $d \geqslant \delta \geqslant 3$ and $x \in\left[\frac{1}{\delta^{3}}, \frac{1}{3}\right]$, then

$$
(1-x)^{d+1}-\frac{1}{1+d} x^{d+1} \leqslant(1-x)^{\delta+1}-\frac{1}{1+\delta} x^{\delta+1}
$$

Proof of Claim 1: We prove the inequality $(1-x)^{d+1}-\frac{1}{1+d} x^{d+1} \leqslant(1-x)^{d}-\frac{1}{d} x^{d}$ for $d \geqslant \delta+1 \geqslant 4$ and $x \in\left[\frac{1}{\delta^{3}}, \frac{1}{3}\right]$. Because of $(1-x)^{d+1}=(1-x)^{d}-x(1-x)^{d}$ this inequality is equivalent to $\frac{1}{d}-\frac{x}{1+d} \leqslant x \frac{(1-x)^{d}}{x^{d}}$. Since $x \leqslant \frac{1}{3}$, we have $\frac{(1-x)^{d}}{x^{d}} \geqslant 2^{d}$ and it is sufficient to show that $\frac{1}{d} \leqslant x 2^{d}$ for $d \geqslant \delta+1 \geqslant 4$. Since $x \geqslant \frac{1}{\delta^{3}}>\frac{1}{d^{3}} \geqslant \frac{1}{d 2^{d}}$, the last condition holds and the proof is complete.

By Claim 1, for $x \in\left[\frac{1}{\delta^{3}}, \frac{1}{3}\right]$ Corollary 4 implies

$$
\gamma(G) \leqslant \frac{n}{2 \delta+1}\left((2 \delta x+1)+\delta\left((1-x)^{\delta+1}-\frac{1}{1+\delta} x^{\delta+1}\right)\right)
$$

and we consider the problem of minimizing this term with respect to $x \in\left[\frac{1}{\delta^{3}}, \frac{1}{3}\right]$.

Therefore, let

$$
h(x)=(\delta+1)(1-x)^{\delta}+x^{\delta} .
$$


Note that

$$
\frac{\partial}{\partial x}\left((2 \delta x+1)+\delta\left((1-x)^{\delta+1}-\frac{1}{1+\delta} x^{\delta+1}\right)\right)=\delta(2-h(x))
$$

Claim 2 The function $h(x)$ is strictly decreasing for $x \in\left[0, \frac{1}{2}\right], h\left(\frac{1}{\delta^{3}}\right) \geqslant 2$ and $h\left(\frac{1}{3}\right) \leqslant 2$.

Proof of Claim 2: Since $\frac{d}{d x} h(x)<0$ is equivalent to $\left(\frac{x}{1-x}\right)^{\delta-1}<\delta+1$ and $\frac{x}{1-x}<1$ for $x \leqslant \frac{1}{3}$, the function $h(x)$ is strictly decreasing for $x \in\left[0, \frac{1}{2}\right]$.

Clearly, $h\left(\frac{1}{\delta^{3}}\right) \geqslant 2$ if and only if $(\delta+1)\left(1-\frac{1}{\delta^{3}}\right)^{\delta}+\left(\frac{1}{\delta^{3}}\right)^{\delta} \geqslant 2$. This can easily be checked for $3 \leqslant \delta \leqslant 5$. For the remaining values of $\delta$ it is sufficient to show that $\left(1-\frac{1}{\delta}\right)^{\delta} \geqslant \frac{2}{\delta+1}$. Since $\left(1-\frac{1}{\delta}\right)^{\delta} \geqslant \frac{8}{27}$ for $\delta \geqslant 3$ this last inequality is true for $\delta \geqslant 6$.

For $\delta=3$ one easily checks that $h\left(\frac{1}{3}\right) \leqslant 2$ and for $\delta \geqslant 4$, we have

$$
h\left(\frac{1}{3}\right)=(\delta+1)\left(\frac{2}{3}\right)^{\delta}+\left(\frac{1}{3}\right)^{\delta}<2(\delta+1)\left(\frac{2}{3}\right)^{\delta} \leqslant 2
$$

which completes the proof of the claim.

By Claim 2, there is a unique $x \in\left[0, \frac{1}{2}\right]$ with $h(x)=2$ which lies in $\left[\frac{1}{\delta^{3}}, \frac{1}{3}\right]$ and the proof is complete.

The following table contains some numerical results concerning the bound in Corollary 5 and the point $x_{0}$.

\begin{tabular}{|r||c|c|}
\hline$\delta$ & bound & $x_{0}$ \\
\hline \hline 3 & 0.4895676537 & 0.2074827860 \\
4 & 0.4344421097 & 0.2049045685 \\
5 & 0.3918579884 & 0.1972824290 \\
6 & 0.3578840276 & 0.1884404884 \\
7 & 0.3300593960 & 0.1796649981 \\
8 & 0.3067865527 & 0.1713933478 \\
9 & 0.2869859624 & 0.1637489730 \\
10 & 0.2699010113 & 0.1567356685 \\
\hline
\end{tabular}

Table 3 Numerical results for Corollary 5

\section{Working along a Random Permutation}

The following very simple probabilistic argument yields a proof of the well-known lower bound for the independence number of a graph due to Caro [4] and Wei [13]. Let $G=$ $(V, E)$ be a graph. For a random linear ordering $v_{1}, \ldots, v_{n}$ of its vertices let

$$
I=\left\{v_{i} \mid N_{G}\left(v_{i}\right) \cap\left\{v_{1}, \ldots, v_{i-1}\right\}=\emptyset\right\} .
$$


Let $v_{i} \in V$. Since every vertex $v_{j} \in N_{G}\left[v_{i}\right]$ appears with equal probability as the first vertex among the $1+d_{G}\left(v_{i}\right)$ vertices in $N_{G}\left[v_{i}\right]$ within the linear ordering, we have $\mathbf{P}\left[v_{i} \in\right.$ $I]=\frac{1}{1+d_{G}\left(v_{i}\right)}$ and hence, by linearity of expectation,

$$
\alpha(G) \geqslant \sum_{v \in V} \frac{1}{1+d_{G}(v)} .
$$

Our aim is to mimic this approach in order to construct dominating sets. A first attempt to do so would be to start with an empty set $D$ and then - following some random linear ordering - to add the vertices of a graph $G$ one by one to $D$ exactly if they have no neighbour in $D$. As in Section 2 the analysis of this approach is difficult, because of accumulating dependencies. We modify the described procedure in such a way that every vertex which still might be useful for dominating a neighbour following later in the linear ordering belongs to the constructed dominating set at least with some small probability. While the mentioned dependencies are still there, this modifications allows an analysis leading to an upper bound.

Theorem 6 If $G=(V, E)$ is a graph of order $n$ and

$$
\rho=\frac{1}{n} \sum_{v \in V} \frac{1}{1+d_{G}(v)},
$$

then

$$
\gamma(G) \leqslant \begin{cases}p^{*} n+\frac{1-p^{*}-\left(p^{*}\right)^{2}}{p^{*}} \sum_{v \in V} \frac{1}{1+d_{G}(v)} & , \text { for } p^{*}=\sqrt{\frac{\rho}{1-\rho}} \text { and } \rho \leqslant \frac{1}{5} \\ p^{* *} n+\frac{1-p^{* *}}{p^{* *}} \sum_{v \in V} \frac{1}{1+d_{G}(v)} & , \text { for } p^{* *}=\sqrt{\rho} \text { and } \rho \leqslant \frac{1}{4} .\end{cases}
$$

Proof: Let $v_{1}, \ldots, v_{n}$ be a random linear ordering of the vertices of $G$. For $1 \leqslant i \leqslant n$ let $N_{i}^{-}=N_{G}\left(v_{i}\right) \cap\left\{v_{1}, \ldots, v_{i-1}\right\}$ be the set of neighbour of $v_{i}$ preceeding $v_{i}$ within this ordering. For some $p \in\left[0, \frac{1}{2}\right]$ we consider the following algorithm

\section{Algorithm $B_{p}$}

1. $D:=\emptyset$.

2. For $i$ from 1 to $n$ do:

$$
\begin{aligned}
& \text { If } N_{i}^{-} \cap D=\emptyset \text {, then } D:=D \cup\left\{v_{i}\right\} \text {, } \\
& \text { If } N_{i}^{-} \cap D \neq \emptyset \text { and }\left|N_{i}^{-}\right|<d_{G}\left(v_{i}\right) \text {, then } D:=D \cup\left\{v_{i}\right\} \text { with probability } p .
\end{aligned}
$$

3. Output $D$.

Clearly, $D$ is a dominating set. Note that for every $0 \leqslant k \leqslant d_{G}\left(v_{i}\right)$ we have

$$
\mathbf{P}\left[\left|N_{i}^{-}\right|=k\right]=\left(\begin{array}{c}
d_{G}\left(v_{i}\right) \\
k
\end{array}\right) \frac{k !\left(d_{G}\left(v_{i}\right)-k\right) !}{\left(1+d_{G}\left(v_{i}\right)\right) !}=\frac{1}{1+d_{G}\left(v_{i}\right)} .
$$


Furthermore, since every vertex $v_{j}$ for which $N_{j}^{-}$does not contain all its neighbour belongs to $D$ with probability at least $p$, we have

$$
\mathbf{P}\left[N_{i}^{-} \cap D=\emptyset|| N_{i}^{-} \mid=k\right] \leqslant(1-p)^{k} .
$$

Therefore, we obtain

$$
\begin{aligned}
& \mathbf{P}\left[v_{i} \in D\right]=\sum_{k=0}^{d_{G}\left(v_{i}\right)} \mathbf{P}\left[\left|N_{i}^{-}\right|=k\right] \cdot \mathbf{P}\left[N_{i}^{-} \cap D=\emptyset|| N_{i}^{-} \mid=k\right] \\
& +\sum_{k=0}^{d_{G}\left(v_{i}\right)-1} \mathbf{P}\left[\left|N_{i}^{-}\right|=k\right] \cdot \mathbf{P}\left[N_{i}^{-} \cap D \neq \emptyset|| N_{i}^{-} \mid=k\right] \cdot p \\
& =\sum_{k=0}^{d_{G}\left(v_{i}\right)} \mathbf{P}\left[\left|N_{i}^{-}\right|=k\right] \cdot \mathbf{P}\left[N_{i}^{-} \cap D=\emptyset|| N_{i}^{-} \mid=k\right] \\
& +\sum_{k=0}^{d_{G}\left(v_{i}\right)-1} \mathbf{P}\left[\left|N_{i}^{-}\right|=k\right] \cdot\left(1-\mathbf{P}\left[N_{i}^{-} \cap D=\emptyset|| N_{i}^{-} \mid=k\right]\right) \cdot p \\
& =\mathbf{P}\left[\left|N_{i}^{-}\right|=d_{G}\left(v_{i}\right)\right] \cdot \mathbf{P}\left[N_{i}^{-} \cap D=\emptyset|| N_{i}^{-} \mid=d_{G}\left(v_{i}\right)\right] \\
& +\sum_{k=0}^{d_{G}\left(v_{i}\right)-1} \mathbf{P}\left[\left|N_{i}^{-}\right|=k\right] \cdot\left(p+(1-p) \cdot \mathbf{P}\left[N_{i}^{-} \cap D=\emptyset|| N_{i}^{-} \mid=k\right]\right) \\
& \stackrel{(8)}{=} \frac{1}{1+d_{G}\left(v_{i}\right)} \cdot \mathbf{P}\left[N_{i}^{-} \cap D=\emptyset|| N_{i}^{-} \mid=d_{G}\left(v_{i}\right)\right] \\
& +\sum_{k=0}^{d_{G}\left(v_{i}\right)-1} \frac{1}{1+d_{G}\left(v_{i}\right)} \cdot\left(p+(1-p) \cdot \mathbf{P}\left[N_{i}^{-} \cap D=\emptyset|| N_{i}^{-} \mid=k\right]\right) \\
& \stackrel{(9)}{\leqslant} \frac{1}{1+d_{G}\left(v_{i}\right)} \cdot\left((1-p)^{d_{G}\left(v_{i}\right)}+\sum_{k=0}^{d_{G}\left(v_{i}\right)-1}\left(p+(1-p) \cdot(1-p)^{k}\right)\right) \\
& =\frac{1}{1+d_{G}\left(v_{i}\right)} \cdot\left(p d_{G}\left(v_{i}\right)+\frac{2 p-1}{p}(1-p)^{d_{G}\left(v_{i}\right)}+\frac{1-p}{p}\right) \\
& \stackrel{2 p \leqslant 1}{\leqslant} \frac{1}{1+d_{G}\left(v_{i}\right)} \cdot\left(p d_{G}\left(v_{i}\right)+\frac{1-p}{p}\right) \\
& =p+\frac{1-p-p^{2}}{p\left(1+d_{G}\left(v_{i}\right)\right)} \text {. }
\end{aligned}
$$

By linearity of expectation,

$$
\begin{aligned}
\gamma(G) & \leqslant p n+\frac{1-p-p^{2}}{p} \sum_{v \in V} \frac{1}{1+d_{G}(v)} \\
& \leqslant p n+\frac{1-p}{p} \sum_{v \in V} \frac{1}{1+d_{G}(v)} .
\end{aligned}
$$

THE ELECTRONIC JOURNAL OF COMBINATORICS 17 (2010), \#R102 
Let $\rho=\frac{1}{n} \sum_{v \in V} \frac{1}{1+d_{G}(v)}$. For the bound in (10) the optimal value for $p$ equals $\sqrt{\frac{\rho}{1-\rho}}$ which is at most $\frac{1}{2}$ for $\rho \leqslant \frac{1}{5}$. Similarly, for the bound in (11) the optimal value for $p$ equals $\sqrt{\rho}$ which is at most $\frac{1}{2}$ for $\rho \leqslant \frac{1}{4}$. This completes the proof.

\section{References}

[1] N. Alon and J. Spencer, The Probabilistic Method, John Wiley and Sons, Inc., 1992.

[2] V.I. Arnautov, Estimation of the exterior stability number of a graph by means of the minimal degree of the vertices, (Russian), Prikl. Mat. Programm. 11 (1974), 3-8.

[3] M. Blank, An estimate of the external stability number of a graph without suspended vertices, Prikl. Math. Programm. Vyp 10 (1973), 3-11.

[4] Y. Caro, New results on the independence number, Technical Report. Tel-Aviv University (1979).

[5] F. Göring and J. Harant, On domination in graphs, Discuss. Math., Graph Theory 25 (2005), 7-12.

[6] J. Harant, A. Pruchnewski, and M. Voigt, On Dominating Sets and Independent Sets of Graphs, Combin. Prob. Comput. 8 (1999), 547-553.

[7] T.W. Haynes, S.T. Hedetniemi and P.J. Slater, Fundamentals of domination in graphs, Marcel Dekker, Inc., New York, 1998.

[8] T.W. Haynes, S.T. Hedetniemi and P.J. Slater, Domination in graphs: advanced topics, Marcel Dekker, Inc., New York, 1998.

[9] W. McCuaig and B. Shepherd, Domination in graphs with minimum degree two, $J$. Graph Theory 13 (1989), 749-762.

[10] O. Ore, Theory of graphs, Amer. Math. Soc. Colloq. Publ. 38, 1962.

[11] C. Payan, Sur le nombre d'absorption d'un graphe simple, (French), Cah. Cent. Étud. Rech. Opér. 17 (1975), 307-317.

[12] B. Reed, Paths, stars and the number three, Combin. Prob. Comput. 5 (1996), 267276.

[13] V.K. Wei, A lower bound on the stability number of a simple graph, Bell Laboratories Technical Memorandum 81-11217-9, Murray Hill, NJ, 1981. 